\title{
Multi-Polygonal Object Tracking
}

\author{
Guido Tascini, Paolo Puliti and Primo Zingaretti
}

\author{
Istituto di Informatica, Facoltà di Ingegneria, Università di Ancona, Italy \\ e-mail: zinga@anvax2.cineca.it
}

\begin{abstract}
In this paper we consider the problem of the recognition and tracking of moving objects from monocular sequences of images. In particular, we describe a new strategy for $2 \mathrm{D}$ intelligent temporal tracking of objects. The approach is feature-based and resolves the correspondence between points in two consecutive images with geometric tracking. While in literature often they are used edges or corners here we use an advanced segmented image, that may be represented with a series of polygons, whose correspondence in consecutive images constitutes the basis of the tracking. The architecture of our vision system is structured on two sub-systems: the 'Static Recognizer', which allows to verify the presence of an object in a scene, and the 'Dynamic Recognizer', which allows to track an object after the relative translation and/or rotation.
\end{abstract}

\section{Introduction}

Correspondence detection and motion estimation are two main topics in image sequence analysis and tracking.

The motion estimation from a series of images is a well described problem in literature $[3,5,6]$. Normally there are two types of approaches: pixel based and feature based. The pixel based, or flow based, methods use local changes in light intensity to compute optical flow at each image point and then derive 3D motion parameters [1]. The feature based methods extract the features (corners, point of curvature, lines, etc.), and use them to estimate the motion [7].

The motion correspondence problem concerns the features in two consecutive frames. Given a frame sequence and $\mathrm{m}$ points in each frame, the motion correspondence maps points between frames such that no two points of a frame map on the same point of the other frame. The problem is combinatorial and may be constrained by some assumptions as the 'proximal uniformity constraint' proposed by Rangarajan and Sah [8].

In this paper we consider the problem of the recognition and tracking of moving objects from monocular sequences of images. In particular, we describe a new strategy for $2 \mathrm{D}$ intelligent temporal tracking of objects. This strategy, adopted in the active vision system developed at our Institute, speeds up the whole object recognition process, and represents a fundamental step towards a real time high level symbolic scene interpretation. Tracking intelligence mainly relies on the integration of $2 \mathrm{D}$ motion estimation results (dynamic knowledge), updated at every frame, directly into the feature-correspondence detection-process: the use of dynamic knowledge greatly facilitates the recognition by reducing both the search space and the algorithm computational complexity. 
Our approach is feature based. The polygonal representation of the regions obtained from the segmentation is used as a basis for the extraction of features, as edges, corners or whole-regions attributes, which can be easily retrieved in the successive frames, forming a correspondence chain. We adopt a geometric matching approach which uses parametrized geometric models for the matching of two point patterns from two consecutive images, and may be viewed as a parametrized optimization problem.

The architecture of our active vision system is structured on two sub-systems: the 'Static Recognizer', which allows to verify the presence of an object in a scene by analysing the information contained in a single frame of a sequence, and the 'Dynamic Recognizer', which allows to track an object after the relative translation and/or rotation produced by its movements in the visual field and/or by a camera moving through the environment.

The paper is organized as follows. In the next section we adduce reasons for a multi-polygonal approach to object tracking. Section 3 describes how acquired images are processed and transformed to obtain the input data for the recognizers, which are described in section 4. Results and conclusions are finally reported in section 5.

\section{Polygonal Approach}

As we will see, our approach to object tracking needs of well segmented images. So fundamental are the choices adopted concerning the environment, the techniques of image pre-processing and the geometric knowledge representation.

Preliminary to object tracking is object extraction. Our approach intends to resolve the problem of object extraction from images by a process of segmentation through multithresholding, and therefore represented from homogeneous regions in intensity. In particular, our vision system adopts a viewer centred representation both for the data image and for the models in the knowledge base. Objects are represented by a number of views (aspects) and are partitioned into regions from each of which the visual features are visible.

This choice involves the object extraction process to be characterized by data transformations in order to arrive at the process of matching among homogeneous representations. In the next section we will describe the structure and the characteristics of a vision system that supports this representation, specifying the transformations of low and intermediate level in the processes of vision.

Despite using regions as primitives at a low level knowledge-representation, we adopt a boundary description at an intermediate-level of knowledge-representation for the regions resulting from the segmentation process [4]. But, for their genesis such contours are different from those obtained through edge representations as there are neither problems of closing or thickness (we easily obtain one-pixel width contours without application of thinning algorithms or similar processing). The principal reason of such choice is related to the semantic meaning of the entities resulting from the segmentation: in the case of edge representation the segmentation process halts to a level of organization before the region representation, which, in a sense, furnishes both edges and regions.

In the edge representation the edges obtained from the segmentation process characterize the separation parts among regions with certainty, but they do not allow 
to characterize regions exactly; this involves a great effort of elaboration in the transformation processes that will conduct to the interpretation of the image.

In the region representation the regions obtained from the segmentation process denote an almost certain clustering of the pixels, even if the certainty decreases toward the boundaries of the regions in the cases of uniform changes of light intensity. The greater computational complexity of this process and the lower reliability of the obtained results are greatly balanced from the simplification that is obtained in the following phases of matching. Obviously the capability of integrating the two representations (data fusion) improves the throughputs of the system simplifying the high level processes.

We perform the segmentation to obtain a region representation, but we also adopt image transformations (image reduction [4]) that conduct to the revision of the primitives at intermediate level (generalized polygonals [9]) in terms of low level primitives (edges). This exploits the analogy with the perceptive abilities of the natural vision systems, which do great use of the edges and of the relations (parallelism, co-linearity, co-ending, etc.) among them.

Again, very important is the influence of the semantic content assigned to an entity in a given representation and, above all, as this semantic value is used in the transformation. For example, in the transformation from a generalized polygonal to a description of the contour through "asperities" [9], assigning the attribute of "gulf" or "peninsula" involves a semantic choice that will be held in consideration in the following processes.

\section{Image Acquisition and Pre-processing}

The implemented algorithms operate on images acquired from three-dimensional scenes in external environment with natural light. This involves treating noisy input data as reflections and diffusions of the natural light on the object surfaces.

The images, acquired from a camera connected to a frame grabber, have a resolution of $512 * 512$ pixels and 256 grey levels. A real time processor performs preprocessing operations: noise removal, spatial filtering, and extraction of image characteristics as intensity histograms and profiles.

The images are segmented using a multithresholding process that selects grey-level intensity-ranges (thresholds) on the basis of local image characteristics. An analysis of these homogeneous connected components is then performed and the resulting labelled regions are 'polygonalized', that is described by a polygonal representation.

In general the polygonals obtained from the segmentation will have a correspondence $n$ to 1 with (the sub-parts of) an object, and in particular they could be in correspondence one to one with (the sub-parts of) an object. In presence of noise, as in our case, also the correspondence $n$ to 1 could be broken and a polygonal could correspond to more than one (sub-part of an) object.

In these situations it becomes important to characterize robust criteria for a segmentation refinement. For example, by using a geometric processor for assigning a probability of merging / splitting (to obtain the set of the regions to match with the model regions), or by using perceptual criteria for driving the grouping process.

In fig. 1 it is sketched the transformation chain from the grabbed image to the input image furnished to the recognizers. 


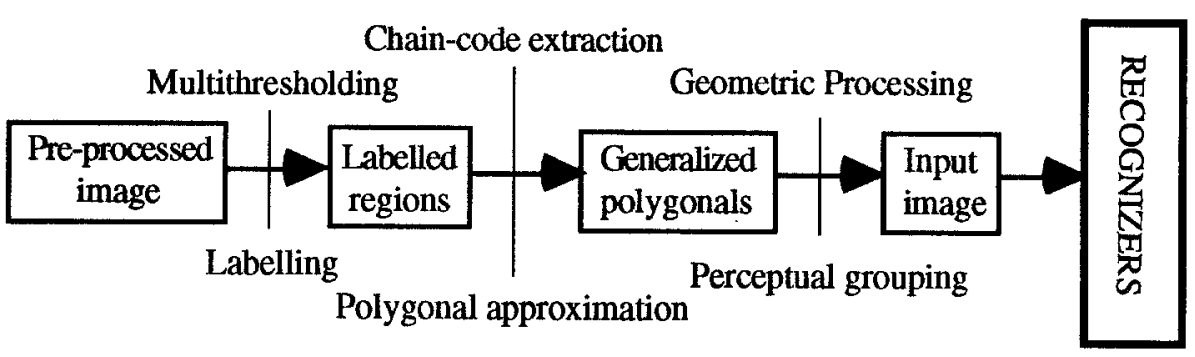

Fig. 1. Transformation chain of the image segmentation process.

\section{The Recognizers}

The general system architecture contains two sub-systems: the 'Static Recognizer' and the 'Dynamic Recognizer'.

Each input image sequence represents a 2D projection of a 3D scene at a given time. Then the matching among the representations of the models and the input data may happen at 2D level. Even if both the recognizers of the system operate at 2D level they have different tasks.

The 'Static Recognizer' is involved in the recognition of a scene, which will result in the selection of the models corresponding to objects which are present in the scene, and, possibly, if they are static or dynamic objects.

The task of the 'Dynamic Recognizer' is the tracking of a 'known' object in a sequence of frames, and involves both the detection of correspondences between successive frames and the motion estimation of the polygonals (objects). The 'Dynamic Recognizer' use a linear model of motion which will be briefly described.

\subsection{The 'Static Recognizer'}

The 'Static Recognizer' is activated in the initial frame and in any other frame where a new ('unknown') object appears in the scene or the Dynamic Recognizer' has failed in tracking a ('known') object.

At present state of implementation, it is responsibility of the user to furnish the 'Static Recognizer' with the models it should recognize in the actual frame. Then, starting from the input image resulting from the segmentation process, and chosen a distinctive polygon (guide-polygon) belonging to the 2D model of the selected object, the 'Static Recognizer' performs a search for similar polygons on the image. The criterion used for establishing the similarity among polygons has been based on the algorithm of Arkin et al. [2]. This search results in a set of capabilities that will be reviewed by considering the knowledge on the complete model. In this phase there are further couplings among polygons (domain dependent grouping), derived both from the model and the input data, which confirm the local matching.

It follows a matching phase which furnishes the scaling, rotation, and translation parameters that allow to superimpose the model to the image data. The aim of this phase is to improve the solution previously found using a simple iterative algorithm. 


\subsection{The Linear Model of Motion}

The motion information is inferred directly from the sequence of images. The object motion is extracted from two successive frames making possible to anticipate the object position in the next frame. The changes of position and of appearance of the regions are due to the combined effect of the relative motion of the object respect to the image plane and of the projection performed by the camera. We use a linear affine model for the motion estimation.

In general we can assume that a region $R$ in the image at time $t+1$ is the result of an affine transformation $f(\phi, u)$ of the image at time $t$, where $\phi(t)$ is the rotation matrix and $u(t)$ is the displacement vector. The points of $R$ at time $t+1$ are located at $(x(t+1), y(t+1))$ and satisfy the relation:

$$
\left(\begin{array}{l}
x \\
y
\end{array}\right)(t+1)=\phi(t)\left(\begin{array}{l}
x \\
y
\end{array}\right)(t)+u(t)
$$

Let $\mathbf{p}$ the position of a point of the object in the frame at time $t$ and $\mathbf{p}^{\prime}$ the correspondent position in the frame at time $t+1$; we could write: $p^{\prime}=\mathbf{f}(\mathbf{p})$ where $f$ is a vectorial linear function. Then to estimate the polygon modifications it will be used the following linear relation, which is characterized from 6 independent parameters (4 for the $S$ matrix and 2 for the $u$ vector):

$$
\mathbf{p}^{\prime}=\mathbf{S} \mathbf{p}+\mathbf{u} \quad \text { where } \mathbf{S}=\left[\begin{array}{l}
S_{11} S_{12} \\
S_{21} S_{22}
\end{array}\right] \text { and } \mathbf{u}=\left[\begin{array}{l}
\mathbf{u}_{\mathbf{x}} \\
\mathbf{u}_{\mathbf{y}}
\end{array}\right] \text {. }
$$

\subsection{The 'Dynamic Recognizer'}

The dynamic analysis of the frames is directed to moving objects, which are hypothesized as previously detected in the static phase and then must be tracked in the dynamic phase.

The 'Dynamic Recognizer' is constituted by three modules: 'Correspondence Detector', 'Motion Estimator' and 'Tracking Controller'.

'Correspondence Detector'. The 'Correspondence Detector' analyses the polygons in the ROIs of two consecutive frames $i$ and $i+1$, and formulates a set of hypothezes about plausible correspondences. The correspondence reliability will be evaluated in terms of indexes (translation index, index of dimension, index of shape, etc.) combined in the aggregate index of plausibility.

'Motion Estimator'. The 'Motion Estimator', using a linear affine model, evaluates the motion parameters by an iterative matching process, giving the values of the 6 parameters that minimize a certain index of disagreement (mismatch index) between the corresponding polygons. This index is constituted by the total area of the two polygons that does not belong to the zone of overlap, after their barycentres has been superposed. The calculus of the geometric intersections among polygons is performed by a 'Geometric Processor'.

'Tracking Controller'. The 'Tracking Controller' is the module supervising the complete tracking operation. It uses the results obtained at frame i from the Motion 
Estimator' for a correct positioning and sizing of the ROI on which the 'Correspondence Detector' will perform the search. Finally it decides for a missing or successful recognition, respectively invoking the 'Static Recognizer' or giving the polygons in the frame $i+1$ that should be tracked in the $i+2$ frame.

\section{Results and Conclusions}

The tracking of multi-polygonal objects was tested on different real frame sequences. A statistical analysis has not been performed yet. Anyway the observations have confirmed as the region representation by generalized polygonals allows an efficient tracking. In particular, our region based tracking allows a very easy matching process, a more accurate estimation of motion parameter (assigned to a dense region) than in optical-flow point based methods, and a good tolerance to noise and partial occlusions.

As the frame acquisition speed, while the 'Static Recognizer' does not require the usual constraints of small motion and smooth intensity profile (it only requires well segmented images), the tracking strategy ('Dynamic Recognizer') requires no too high rate, sufficient to keep limited the changes between two consecutive frames.

Furthermore, this strategy may efficiently be implemented on parallel machines, assigning the tracking of each object part (or multiple target objects) to independent processes and the overall control to another process.

\section{References}

1. Adiv G., "Determining Three-Dimensional Motion and Structure from Optical Flow Generated by Several Moving Objects", IEEE Trans on PAMI, vol. 7, no. 4, pp. 384-401, 1985.

2. Arkin E.M., Chew L.P., Huttenlocher D.P., Kedem K., Mitchell J.S.B., "An Efficiently Computable Metric for Comparing Polygonal Shapes", IEEE Trans. on PAMI, vol. 13, no. 3, pp. 209-216, 1991.

3. Broida T.J., Chellappa R, "Estimation of object motion parameters from noisy images", IEEE Trans. on PAMI, vol. 8, no. 1, pp. 90-99, 1986.

4. Giretti A., Puliti P., Tascini G., Zingaretti P., "Segmented Image Reduction", Intelligent Perceptual Systems, V.Roberto ed., Lectures Notes in Artificial Intelligence, Springer-Verlag, pp. 181-194, 1993.

5. Jain R., "Segmentation of frame sequences obtained by a moving observer", IEEE Transaction on PAMI, vol. 6, pp. 624-629, 1984.

6. Kalivas D.S., Sawchuk A.A., "A Region Matching Motion Estimation Algorithm", CVGIP, vol. 54, no. 2, pp. 275-288, 1991.

7. Lee C.H., "Interpreting image curve from multiframes", Artificial Intelligence, vol. 53, pp. 145-163, 1988.

8. Rangarajan K., Shah M., "Establishing Motion Correspondence", CVGIP: Image Understanding, vol. 54, no. 1, pp. 56-73, 1991.

9. Tascini G., Puliti P., Zingaretti P., "Segmentation Suggested Geometric Scheme", Proc. SPIE 1832, pp. 280-286, 1992. 